Marcello Ciccuto

\title{
Francesco da Barberino e la parola delle immagini nei Documenti d'Amore
}

\author{
Antefatti del «visibile parlare»
}

\begin{abstract}
In questo saggio cerco di mostrare come nei molteplici rapporti intrecciati alle parabole culturali di Dante Alighieri, Francesco da Barberino e Giotto si sia venuta definendo agli inizi del Trecento una nuova retorica del visibile, giocata su allegorie, personificazioni di vizi/virtù e transumptiones che la scrittura letteraria fa proprie attraverso il confronto costante con cicli figurativi e illustrazioni librarie. La sintesi funzionale tra le valenze cognitive di scritture e figure viene studiata attraverso la lettura congiunta di alcuni aspetti decisivi nel processo di definizione di una nuova visual literacy, che riguardò contemporaneamente i Documenti d'Amore del Barberino, la Cappella degli Scrovegni affrescata da Giotto e il concetto di «visibile parlare» centrato da Dante nei canti X-XII del Purgatorio.
\end{abstract}

This essay demonstrates how, driven by the outstanding cultural figures that were Dante Alighieri, Francesco da Barberino and Giotto, the early fourteenth century in Italy developed a new "rhetoric of the visible", playing with symbols, personifications of vices/virtues and transumptiones which literary writing appropriated in constant view of figurative cycles and book illustrations. The functional synthesis of the cognitive dimensions of writings and figures is analysed against the backdrop of a new visual literacy which concerned, at the same time, Barberino's Documenta Amoris, Giotto's fresco cycles in the Scrovegni Chapel and Dante's concept of „visibile parlare“ (Purgatory, cantos X-XII).

Parole chiave: Dante Alighieri, Giotto, Francesco da Barberino, Documenti d'Amore, Cappella degli Scrovegni, Visibile parlare

L'ambiente padovano di primo Trecento risulta percorso da quasi infinite e ancora non perfettamente inquadrabili tensioni, addensate specialmente e con particolare vicinanza al discorso che stiamo per fare attorno alle imprese della bottega giottesca all'Arena Scrovegni e quindi nel Palazzo della Ragione in rapporto a quanto Francesco da Barberino fu in grado di mostrare sui fogli delle sue opere.

Entro una fittissima rete di incroci e scambi di competenze, spesso diretti da personalità di grido - da Pietro d'Abano a Francesco medesimo e quindi a Giotto e alla sua bottega - che si rivelano implicate in un vasto processo di ride- 
finizione di una "visualità" che sulle carte di libri illustrati o sulle pareti di edifici pubblici e privati si era proposta di elaborare i codici collaborativi fra testi letterari e immagini della tradizione tardo-classica in una chiave che, entro un'ormai comprovata «attitudine a pensare per immagini», ${ }^{1}$ potesse soddisfare un'esigenza tutta laica di affidare proprio alle immagini funzioni didattiche nuove e più “aperte" rispetto al passato, parecchi operatori si trovarono impegnati a elaborare per il pubblico affluente di quell'area un "vedere" che dentro gli antichi schemi di percezione allegorica del mondo potesse far fruttare prospettive meglio aggiornate: ad esempio al fuoco di esperienze tanto varie quanto registrate su dotazioni e portati intellettuali che oggi definiremmo "di nuova generazione” (fossero essi quelli convogliati sul territorio dall'arrivo di manoscritti illustrati di materia astrologica più avanzata rispetto al passato classico, lo sviluppo e il radicamento di una pubblicistica giuridica di respiro internazionale o le sirene che dai vicini "giacimenti" di Avignone e della dotta Bologna facevano sentire a più livelli il proprio richiamo assieme a una forza modellizzante fino a quel momento impensata sul piano schiettamente culturale). ${ }^{2}$

La realizzazione da parte del Barberino, proprio a Padova nel quinquennio 1304-1308, dell'oggi recuperato Offiziolo ${ }^{3}$ permette di incrociare subito alcuni

1 Così Mariani Canova, L'“Officiolo”, p. 70.

2 Per un panorama ampio e dettagliato nonché misurato in specifico su questa importante area culturale - pur in mezzo a una bibliografia che è possibile avvicinare qui solo in iscorcio si vedano almeno Toniolo, Il libro miniato, Banzato, L'impronta di Giotto, Romano, Giotto e la nuova pittura e Romano, La O di Giotto. Quanto alle collaborazioni di testi e immagini all'interno della prima poesia volgare, di cui si dirà a breve, cfr. il disegno tracciato in extenso in Ciccuto, Icone della parola e in Mariani Canova, Il poeta e la sua immagine. Più in specifico, una prospettiva generale dei fenomeni che si vedranno coinvolti anche nell'affermarsi di una "cultura astrologica" in questo e in altri contesti collegati si trova in Ciccuto, Figure di Petrarca, Toniolo, Il libro miniato, Blume, Michael Scot. La vicenda del codice dell'opera di Michele Scoto illustrato con immagini provenienti sia dalla tradizione degli Aratea sia da quella orientale del Georgius Zothorus Zaparus Fendulus, forse salito al Nord-Italia nel pieno degli anni '20 del Duecento ed entrato nella compagine del De imaginibus di Pietro d'Abano, è quella che permise a Giotto e aiuti di imporre l'uso di immagini planetarie a piena figura o di solenni icone assise e stanti - pure dentro un lavoro di acquisto a livello stilistico degli effetti "scultorei" della monocromia che avrà un peso tutt'altro che trascurabile nel corso della pittura di taglio umanistico del '300-'400 - delle quali oggi è dato aver memoria solo in base a quanto più tardi Guariento agli Eremitani e infine Miretto e Stefano ferrarese al Palazzo della Ragione lasciarono in vista di quegli antichi radicali. Alla bibliografia specifica indicata poco sopra riguardo a un episodio come questo decisivo per la nascita della "nuova figurazione" di matrice giottesca si può aggiungere oggi anche Blume, Haffner, Metzger, Sternbilder des Mittelalters.

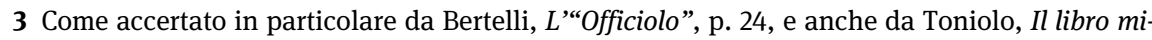
niato, pp. 114-117. 
elementi che serviranno a definire in modo ci auguriamo compiuto il più vasto $\mathrm{e}$ successivo progetto di composizione e illustrazione dei Documenti d'Amore, autentico banco di prova non solo per le competenze a quel punto assai mature dell'autore in materia di Bildlichkeit, ma anche luogo di affermazione di uno statuto del "figurare" e dell'identità dell'artista-letterato che avrebbe svolto grande funzione mediatrice e di accompagnamento a quelle che saranno presto le esperienze dantesche e boccacciane su questo stesso terreno, della riflessione cioè attorno al collaborare di immagini e di parole all'interno della scrittura letteraria. ${ }^{4}$

Mi riferisco, per cominciare, alle più e meno esplicite derivazioni, nel libro d'ore barberiniano, dalla conoscenza del ciclo figurato agli Scrovegni, ${ }^{5}$ da condividersi certo con quanto il maestro di scuola bolognese là operante - sia esso o no il leggendario "Gherarduccio" - riesce a mettere in mostra nell'esteso ciclo dei sei Antifonari notturni, responsoriali festivi e feriali secundum consuetudinem romanae curiae realizzati per la Cattedrale di Padova, ${ }^{6}$ indubitabilmente lavorati in presa diretta, potremmo dire, con l'osservazione dei portati giotteschi all'Arena, e dunque anteriormente a un'evoluzione, sempre e comunque registrata sugli sviluppi patavini dello stesso maestro mugellese, che produrrà con altri artisti di scuola bolognese esecuzioni finanche seriali, pronte a loro volta a riflettersi nel profondo della cultura figurativa barberiniana, come i Lezionari della Cattedrale (manoscritti A 18, A 19, A 20, B 12, B 13 della Capitolare), o l'Evangelistario e l'Epistolario (mss. C 30 e C 31 della stessa Biblioteca); e ancora

4 Per la cultura visiva di Dante e i numerosi esempi che nella Commedia la riguardano basti il richiamo a Ciccuto, Saxa loquuntur, che riattiva altri suoi precedenti interventi. Così, quanto al formarsi di un'identità artistica all'altezza delle esperienze allargate di Boccaccio, è opportuno guardare a Ciccuto, Il novelliere e Ciccuto, Un'antica canzone di Giotto.

5 Cfr. Toniolo, Il libro miniato, in part. pp. 113-115.

6 Per questi Antifonari (mss. B 14, B 15, A 15, A 16, A 18 e A 19 della Biblioteca Capitolare di Padova) cfr. ancora Toniolo, Il Maestro degli Antifonari di Padova e Mariani Canova, La Cattedrale di Padova. Il percorso stilistico del loro operatore principale, se di Gherarduccio si tratta, meno interessato a inquadrature architettoniche di modello giottesco che non a più moderni effetti di coinvolgimento dell'osservatore tramite risorse di evidentia visiva, può essere tracciato a partire da un ambientamento interno alla tradizione dei codici di diritto - di cui ha forte memoria, trattandosi di un creato delle scuole bolognesi: cfr. Toniolo, Il Maestro degli Antifonari di Padova miniatore, pp. 102-105, e Ponchia, Le fonti illustrative, pp. 115-116 - che viene presto ad allinearsi in parallelo a quei radicali centro-italici di cui si discute per le personalità raggianti di un Maestro di Gerona o di un Jacopino da Reggio, per cui si vedano le estese analisi in Ciccuto, Fatti romani e Hoffmann, Die Bibel von Gerona. Per le tappe successive, che vedono il miniatore sciogliere in più distesi effetti di narrativizzazione gli acquisti iniziali sulle carte ad esempio del Roman de Troie oggi a San Pietroburgo o della Miscellanea in volgare della Biblioteca Riccardiana (ms. 1538) - si vedano ancora e ampiamente Toniolo, Il Maestro degli Antifonari di Padova miniatore e Stolte, Der Maestro di Gherarduccio. 
più tardi, e non prima comunque della data del 1321, come un altro esito alto di quella effervescente cultura improntata da Giotto quale fu la Commedia dantesca del codice Egerton 943 della British Library, sul quale dovremo tornare a discutere in ragione delle componenti di "retorica visiva" che vedremo compartecipate così da Francesco come dall'Alighieri. ${ }^{7}$ Sul primo radicarsi del lessico giottesco a Padova si vedrà dunque far conto Francesco da Barberino, pronto ad esempio a ricostruire dentro l'Offiziolo figure e composizioni già agli Scrovegni, ${ }^{8} \mathrm{o}$ a ricordare in esplicito il magistero giottesco in luoghi dei Documenti impegnati nell'usuale argomentare attorno alla funzione delle figure (là dove soprattutto avremo modo di riconoscere a Giotto, al Barberino e anche a Dante la partecipazione a un processo di rifondazione dei significati del figurare allegorico e della personificazione di vizi e virtù attivato addirittura già sul sodo della riforma gregoriana delle immagini). ${ }^{9}$

Se preso insieme all'adozione matura da parte del poeta di figurae vicinissime a quelle introdotte sui banchi giotteschi - e subito, pare, dirottate sulle pareti del Palazzo della Ragione - dal potente afflusso in Padova di una cultura figurativa di taglio astrologico, ${ }^{10}$ anche lo scorgere nel tempo lo scambio consultivo attorno a certe immagini deposte su carte barberiniane - dalla figura mostrata a Baldo da Passignano per la composizione sua di un trattato De spe a ciò che immaginiamo effettuale dietro al prestito di una copia dei Documenti a un personaggio coinvolto in faccende d'arte quale Geri d'Arezzo ${ }^{11}$ - andrà collegato in concreto alla dichiarata coscienza autoriale più volte esplicitata dal Barberino e pure "in figura", vuoi sulle carte dell'Officiolo, vuoi negli stessi Documenti, ${ }^{12}$ dove in particolare questi si raffigura nella veste dello scriptor capace di accogliere il verbo di Amore ai piedi della Rocca («operis huius collector franciscus»); e che, quasi dantescamente av-

7 Su questo codice, dopo gli assaggi di Nardi, Le illustrazioni, cfr. ora la completa disamina di Pegoretti, Indagine su un codice dantesco.

8 Cfr. Toniolo, Il libro miniato, pp. 114-115, e Goldin Folena, Dall'“Officiolo”, pp. 110 sgg.

9 Così in Romano, Giotto e la nuova pittura, pp. 9-10; Romano, La O di Giotto, pp. 221-222; Goldin Folena, Dall'“Officiolo”, pp. 111-113.

10 Per cui cfr. almeno Ciccuto, Figure di Petrarca, pp. 25 sgg., e pure Toniolo, Il libro miniato, pp. 116-117.

11 Per Baldo da Passignano cfr. rapidamente Mariani Canova, $L$ '“Officiolo”, pp. 67-68 o anche Marruzzo, Composizione e significato, pp. 234-235. Sull'importanza del rapporto con Geri d'Arezzo, impegnato addirittura in una sorta di smontaggio delle componenti allegoriche del figurare barberiniano in favore di più decise accentuazioni in questo di evidenze retoricamente segnate, si vedano le ricerche confluite in Geri d'Arezzo, Lettere e dialogo d'amore e in Schirg, "In bivio". Per altro verso cfr. Ciccuto, Francesco da Barberino, p. 92.

12 Mariani Canova, L'“Officiolo”, pp. 80-81, per la c. 3v dell'Offiziolo e la c. 8r del ms. Barb. lat. 4077. 
viato dallo stesso dio a un compito di registrazione "con due penne" - la latina e la volgare - di questa diretta ispirazione, ${ }^{13}$ si presenta in una veste rosata che lo accosta alle funzioni di un'Industria ${ }^{14}$ resa attraverso la precipua virtù dell'eloquenza (poi collocata strategicamente a fianco del sire Amore in giunzione con Cortesia).

Dunque Francesco si riconosce impegnato in un progetto di argomentazione attorno al canone dei vizi e delle virtù, tale da far ricorso sì a figurae ideate e talora realizzate di sua mano ma, insieme, a una scriptura aperta a più significazioni in grazia di una stretta collaborazione interpretativa e adiacenza anche spaziale con queste immagini, scelte al fine di offrire una rinnovata veste metaforica ai concetti in gioco e dunque trasformate direttamente in Bildbegriffe (o immagini-concetto). ${ }^{15}$ È un progetto che ha solide basi, già identificabili nel modello operato da Alain de Lille nell'Anticlaudianus e nel De planctu Naturæ sulla via di una definizione di alcune "metafore iconiche" - de facto personificazioni - alle quali poter affidare un potenziamento di visibilità o evidentia per una scrittura poetica resa così "vivente" almeno quanto incaricata di attraversare l'apparenza superficiale delle immagini/personificazioni e di giungere al più pieno senso dei valori divini dell'esistenza. ${ }^{16}$ Grazie alla suggestione delle nuove forme in immagine la poesia diventa in teoria lo strumento più perfezionato e complesso ai fini di una conoscenza del divino, della più profonda realtà del mondo celata dietro le apparenze visibili: frutto di tutto un modus ymagina-

13 Panzera, Francesco da Barberino, pp. 71-72, e quanto allo stimolo portato verso gli abitanti della Fortezza di Amore nella direzione di un rinnovamento, di un'evoluzione verso un ordo esistenziale ispirato a perfezione cfr. MacLaren, Shaping the Self, pp. 74-75. A segnalare ripetutamente «l'orgoglio del Barberino per l'originalità delle proprie invenzioni» (Goldin Folena), oltre ai richiami sparsi nei Documenti d'Amore in ragione delle precedenti realizzazioni di Francesco funzionali alla stesura del conclusivo trattato allegorico, varrà specialmente il passo in $D A$ (Egidi), III, pp. 286-287, nel quale è parola di quella che dovette essere la più "impegnata” (seppur perduta) tra le miniature del libro d'ore, cioè a dire il Finale Iudicium, oltre a tutte le altre miniature rivendicate a sé dall'autore: per queste, Mariani Canova, L'“Officiolo”, pp. 66-67; Sciacca, Pacino di Bonaguida, p. 200, e Labriola, L'eredità di Giotto, pp. 195-196.

14 DA (Egidi), II, p. 5. E cfr. allora Marruzzo, Composizione e significato, pp. 221-222, e per il valore “di eloquenza” degli ornata purpuramentis, Vasiliu, L'économie de l'image, pp. 271-272. 15 Belting, Das Bild als Text, p. 45.

16 Galand, Les «beaux» signes, pp. 30-33. A corredo della sua interpretazione finalizzata allo studio specifico dell'Offiziolo, pone l'accento sulla funzione modellizzante di Alain anche Goldin Folena, Dall'“Officiolo", pp. 121-122, per poi estendere il discorso e la dimostrazione anche ai Documenti, pp. 130-134. E cfr. anche Marruzzo, Composizione e significato, p. 226. Sul valore delle allegorie quali speaking pictures, e in particolare nella prospettiva di ancora troppo meccaniche personificazioni del testo nella fase del Reggimento si guardi ad Harding, Speaking in Pictures, pp. 30-33. 
lis che, rendendo più viva e vivente la scrittura poetica, ${ }^{17}$ ne accentua la capacità di ascendere i diversi gradi di conoscenza delle forme superiori ed esemplari giusto nel momento in cui la parola potenziata dall'immagine punta a sostenere la ricerca umana di una conformità con sé stessi e, al di là della barriera ontologica, nientemeno che con gli esemplari eterni. ${ }^{18}$

Ma se l'esempio di Alain effettivamente viene a rinsaldare l'idea di uno "sfondamento" del mezzo figurativo sul fronte dei registri retorici dominanti, in un contesto che comincia a prospettare per poesia e letteratura nuove possibilità interpretative del mondo visibile, ${ }^{19}$ resta vero che all'ingegno del Barberino, già predisposto a comprendere la potenza del verbo d'Amore all'interno della ymaginatio (e quindi pronto a sostenere la funzione delle figurae quali accompagnamento alle scripturae, sul piano di quello che l'autore considera, per somma di evidenze, il primo grado di conoscenza delle forme esemplari), ${ }^{20}$ il gigantesco progetto di visualizzazione della “storia dell'uomo nel mondo a ridosso del sacro", attivato dall'operare giottesco all'Arena e sulle pareti del Palazzo della Ragione padovano, porta o addirittura impone l'esigenza di una collaborazione fra i due linguaggi, il poetico e il figurativo, sulla quale l'immagine della Circumspectio figura di un "vedere" portato al massimo grado di intelligenza grazie alla sintesi funzionale di figure e scritture - pone un sigillo di alta coscienza critica. ${ }^{21}$ Barberino rappresenta in modi complessi quella nuova via interpretativa, del mondo visibile e non, che prima appunto con Giotto e quindi specialmente con Boccaccio mostrò di legare l'identità dell'artista "moderno" a tutti i processi intesi a scoprire il vero al di là delle apparenze mondane e delle illusioni della mimesi, secondo

17 Cfr. per questo Prandi, Teologia come pittura, pp. 103, 112-13, mentre per i processi di creazione di images fictives vale quanto accertato da Vasiliu, L'économie de l'image, pp. 261-264.

18 Sui modi e la necessità, in Barberino, di ricorrere continuamente e alla glossa e all'immagine in ragione del fatto che solo attraverso di essi il testo può diventare plurisenso cfr. ancora Panzera, Francesco da Barberino, pp. 41-43.

19 Tale gigantesco processo, al quale non possiamo che accennare qui, è stato da me ricostruito nei vari suoi aspetti in Ciccuto, Figure d'artista e Ciccuto, Un'antica canzone di Giotto; quindi piegato alla circostanza barberiniana in Ciccuto, Francesco da Barberino. E cfr. in ogni caso anche Smout, Sprechen in Bildern, pp. 109 sgg. Andrebbe altresì tentata una ricerca apposita sul nuovo modo di guardare alla realtà non più in termini di specchiamento e di mimesi bensì di integrale trasformazione immaginativa di essa: dunque puntando ad analizzare il discorso barberiniano disteso in più luoghi contro l'illusionismo mimetico, pure nei passaggi attraverso i temi dello specchiarsi frequenti nei Documenti o la discussione attorno alla funzione degli specchi magici (per cui cfr. almeno Panzera, Francesco da Barberino, pp. 201-202). Ciò che mi propongo di realizzare in un contributo prossimo alla stampa.

20 Vasiliu, L'économie de l'image, p. 275.

21 Cfr. Ciccuto, Francesco da Barberino, p. 87; Goldin Folena, Dall'“Officiolo”, pp. 113-117; Panzera, Francesco da Barberino, pp. 41-42. 
quanto ho già ampiamente argomentato altrove; e facendosi inoltre carico di un'identità per l'operatore culturale che sapesse far convergere su un terreno di comune esercizio le funzioni di pictura e di scriptura, l'auctor che Francesco rappresenta addirittura all'opera dentro lo spazio testuale, nell'atto medesimo di costruire e istoriare e far vivere i figuranti del proprio libro, ${ }^{22}$ arriva a coincidere con chi si era proposto definizione verbale e ordinamento visivo dei tanti princìpi morali astratti che regolavano il vivere umano, e che venivano adesso alla portata di una parola poetica potenziata esattamente dal supplemento delle immagini. ${ }^{23}$

Non fu solo questione di legarsi a una prospettiva di cosmografia figurale, entro la quale - tanto per fare un esempio fra i molti - i diagrammi circolari potevano definire entro confini di perfezione e di eternità qualsivoglia "accidente" della fisica terrestre, insomma ognuna delle varie species rerum. ${ }^{24} \mathrm{Si}$ attivò quantomeno un più ampio e generale progetto di svecchiamento dell'antico discorso allegorico-didascalico (proprio ancora delle pagine del Reggimento, più inclini a una sterile sottigliezza d'esercizio della verbalità argomentativa, affidata in prevalenza alle prosopopee delle singole virtù, «ciascuna responsabile del discorso nella sua parte» $)^{25}$ inteso a proporre forme di una fantasmagoria allegorica di nuovo conio rispetto, ad esempio, a quella del pur idolatrato esempio del $D e$ planctu Naturae, fondata su potenzialità retoriche della scrittura cui le immagini ora potevano offrire il soccorso di un plus di evidentia: su un quadro insomma entro il quale glosse e figure materialmente presenti nello spazio testuale agivano ogni funzione informativa e di "metaforizzazione" dell'intero cosmo. ${ }^{26}$

La decisa attenzione alle potenzialità di retorica evidentia riconosciute alla scrittura è quella che d'altronde viene a modellarsi sui sistemi ordinativi del testo operati nell'ambito dei commentari giuridici, come è stato più volte notato

22 Cfr. Harding, Speaking in Pictures, p. 32. Ma vedi anche la figura del Franciscus a c. 8r di DA (Barb. lat. 4077), già collegata da Mariani Canova, L'“Officiolo”, p. 80 al Giovane studioso dell'Offiziolo, c. 3v; per non dire dello scriptor che presiede alla grande miniatura della Rocca di Amore sia in Barb. lat. 4076 che in Barb. lat. 4077 - per cui si veda Goldin Folena, Dall'“Officiolo", p. 146.

23 Marruzzo, Composizione e significato, p. 224.

24 Vasiliu, L'économie de l'image, pp. 264-270, e anche Rodrigues, Nature et connaissance, p. 171. Mentre per il discorso barberiniano relativo alle funzioni ordinative della lega Text und Bild buone osservazioni ha il saggio di Minnis, Amor and Auctoritas.

25 Panzera, Francesco da Barberino, p. 45 e nota 102. Ma vale in particolar modo l'esempio ricordato e discusso da Goldin Folena, Dall'“Officiolo”, pp. 130-135, nonché luoghi affini a DA (Egidi), VI, p. 85 o 227.

26 Sull'esempio precedente di tale prospettiva, ammirato dallo stesso Barberino in Alain, cfr. Goldin Folena, Dall'“Officiolo”, pp. 133-134; Marruzzo, Composizione e significato, p. 233. 
da una parte della critica; ${ }^{27}$ ed essendo cura dello stesso Barberino porre al centro della sua discussione sul valore delle virtù proprio la figura della Giustizia, in unione alla forza equilibratrice dei dettami (e delle figure, anche qui) di una Prudenza dai tratti di radice scopertamente giottesca ${ }^{28}$ e di una Eloquenza posta assieme a Cortesia a fianco di Amore, ${ }^{29}$ una delle ragioni del concreto approdo barberiniano a una moderna narrazione per immagini emerge per somma di prove e di indizi da un'antica discussione sulle misure "equilibrate" del dire e del fare e del figurare che, partita da un confronto in tenzone tra Bonagiunta da Lucca e Guido Guinizzelli, aveva investito direttamente i molti temi legati alla composizione di un "trattato d'Amore" di marca guittoniana e non stilnovistica, capace di porre le nuove potenze significanti delle immagini a disposizione del verbo d'amore, e dunque di avvicinare una innovativa proposta di "visualità" che covava da tempo nella cultura dell'epoca (pur se avversata da una parte consistente della rimeria stilnovistica, come ho avuto modo di dimostrare altrove), ${ }^{30}$ e che adesso si rendeva disponibile per il registro verbale al fine di renderlo - grazie a dotazioni di evidentia ben superiori alle precedenti - il più completo fra gli instrumenta interpretandi. ${ }^{31}$

Oso immaginare che ai rapporti con l'ambiente dei poeti toscani espatriati in Veneto - e pure col Lapo Gianni degli anni fiorentini - si dovesse il definirsi delle posizioni del Barberino in merito alla disputa poematica sugli aspetti figurati di Amore, ora nei termini della composizione della canzone Io non descrivo in altra guisa quale spettacolo “esposto”, ora nell'affinità con le proposte guitto-

27 Ad esempio Panzera, Francesco da Barberino, pp. 32-33, 41-43, o anche p. 69 e nota 15 per il rapporto di Francesco con uno specifico esemplare di Digestum figurato; e Mariani Canova, L'“Officiolo”, p. 92, nonché Goldin Folena, Dall'“Officiolo”, p. 136. Quanto ai valori spaziali tipici del cosiddetto textus inclusus (quale effettivamente riusciamo a riconoscere sulla conformazione di base delle pagine stesse dei Documenti d'Amore) si veda di nuovo Harding, Speaking in Pictures, p. 30.

28 Carapezza, Funzioni digressive, pp. 112, 115.

29 Marruzzo, Composizione e significato, pp. 221-222.

$30 \mathrm{Su}$ questo aspetto ogni elemento è reperibile in Ciccuto, Icone della parola, pp. $13 \mathrm{sgg}$. Per il coinvolgimento di Guittone all'interno di questa nevralgica disputa di valore meta-poetico cfr. anche Panzera, Francesco da Barberino, pp. 34-38, assieme al riesame operato in Guittone d'Arezzo, Del carnale amore. Di qui l'importanza dei fittissimi e continui riferimenti testuali, all'interno dei Documenti, che contengono rinvii più e meno espliciti a questa specifica tenzone fra Bonagiunta e Guinizzelli, e che sto raccogliendo per discuterne in apposito contributo.

31 L'associazione a livello di figurazione per le cobbole e la canzone volgare che chiude il Tractatus amoris del Barberino (cfr. Marruzzo, Composizione e significato, p. 243), incaricati entrambi della funzione didascalica destinata a un affresco, sostengono per parte loro il discorso della più generale e conseguita equivalenza di pictura e di scriptura, per alcune articolazioni della quale vedi ancora gli esempi portati da Goldin Folena, Dall'“Officiolo”, pp. 148-150. 
niane del "Trattato d'Amore", ora nel costante rinvio alla collaborazione di glosse e immagini all'interno dei testo dei Documenti. ${ }^{32}$ La raggiunta complementarietà teorica fra Parola e Figura eseguita dal poeta d'amore diventa nelle pagine del maggiore trattato barberiniano la garanzia di una rinnovata relazione fra uomo, divino e ordine del cosmo naturale: all'insegna di quelle regole di proportio e di habitudo che legano ora le artes verbali e figurali alla verità suprema della translatio theologica, l'intera descrizione del condizione mondana quale effetto del lavoro congiunto di parole e di immagini viene a delineare un percorso virtuoso per l'uomo che, riconosciuto il degrado della temporalità di Natura, può puntare ad intuitum celestium formarum, e dunque alla riappropriazione della "fisica naturale" entro un ordo universitatis che è pegno di eternità e salvezza quanto più le immagini restituiscano la loro sostanza di essenze fatte materia visibile. ${ }^{33}$

Nei diversi luoghi nei quali il Barberino teorico del suo proprio linguaggio mette in mostra le potenze descrittive della parola poetica ora apprese ai sommi concetti astratti di virtù e di vizi, risulta evidente il progetto di accostare nell'insieme dei Documenti quelle picturae che, sciogliendosi in un rapporto appunto di isomorfismo significante col testo, siano in grado di portare addizioni di senso alle parole medesime, contenuti non raggiungibili col solo (e limitato) mezzo verbale, che appunto si tiene per convenzione alla superficie d'apparenza dei fenomeni umani senza riuscire - a questo punto direi per insufficienza rispetto alla forza delle allegorie figurate sulle pagine del trattato - nell'impresa di aiutare l'uomo nell'itinerario di ritorno verso Dio, verso le Sostanze Eterne, verso la Ve-

32 Per tutto quello che Lapo Gianni può aver contribuito su questo percorso (dall'immagine della donna dipinta nella mente/nel cuore al trarre esempio dal mirabile modello che essa personifica - Dolc'è 'l penser che mi notrica 'l core, vv. 5-7 - alla "scrittura" sul libro d'Amore, vv. 25-28; dalla preghiera della mente che arriva figurata davanti alla donna - Donna, se 'l prego de la mente mia, vv. 1-4, ad Amore che si presenta sia come pictor nella mente dell'innamorato sia come compositore diretto delle parole di una ballata - Angioletta in sembianza, vv. 25-27 e Ballata, poi che ti compuose Amore, vv. 1-2; fino alla straordinaria circostanza della canzone Amor, nova ed antica vanitate dove il poeta, con fare da notaio e questionatore, descrive le diverse figure di Amore) rimando ai testi convenientemente raccolti e commentati in Marti, Poeti del dolce stil nuovo. Si tenga in conto comunque di quel che deve aver pesato sull'immagine del testo barberiniano anche il lavoro su uno specifico Decretum Gratiani da parte del Maestro degli Antifonari di Padova, e a seguire sulle carte del "dantesco" Egerton 943, nei termini ad esempio valutati da Toniolo, Il Maestro degli Antifonari di Padova miniatore, pp. 90-102. Quanto ai vari casi di responsabilità d'autore considerati nel Barberino da un punto di osservazione anche giuridico, cfr. almeno MacLaren, Shaping the Self, pp. 86-88.

33 Cfr. Rodrigues, Nature et connaissance, pp. 173-174; Amri-Kilani, Connaissance sensitive, pp. 193-200; Rouillé, L'hymne e specialmente Fuehrer, The Cosmological Implications, pp. 344 sgg. Per il ricercato isomorfismo ontologico già messo a testo in Guglielmo di Conches rinvio senz'altro a Gross, The Cosmology of Rhetoric, pp. 39-53. 
rità nascosta sotto quelle apparenze che restano per il mondo della verbalità nient'altro che segnali sparsi di una spesso irrimediabile inordinatio o dissimiglianza (e che invece queste "figure di perfezione" delle Virtù proposte nelle elaborate immagini dei Documenti si incaricano di annullare entro una traduzione visibile, un'esibizione addirittura delle più alte cognizioni morali). ${ }^{34}$

È possibile intendere oggi che, nei tanti momenti nei quali Francesco da Barberino pone l'esigenza del figurare fianco a fianco alla sua scrittura, sta aprendo il linguaggio del tempo suo alla possibilità di significare anche per imagines il percorso umano verso la Verità, così come verso una recuperabile simiglianza in figura dell'uomo con Dio; ${ }^{35}$ all'unisono, riconoscere un obiettivo cognitivo "pesante" come quello del puntare alla verifica visuale delle forme eterne calate nelle immagini significò fondare una conoscenza del mondo non più vincolata alle sterili apparenze terrene - magari veicolate dal solo mezzo verbale - bensì orientata alla conquista di una aeternitas ora sostanziabile con l'usus delle virtù, come dire identificata nell'impegno attivo dell'uomo nel mondo che ispira la sua azione proprio ai modelli figurati di virtù proposti anche dal Barberino. Si trattò insomma di avanzare il concetto nuovo di una Etternitate - come sappiamo descritta, argomentata e dipinta su un foglio dei Documenti ${ }^{36}$ - non più riflessa nelle illusioni del vivere, in specchiamenti mimetici con la natura terrestre o nelle trascorrenti apparenze della temporalità umana, ma riconoscibile invece nelle immagini di virtù che presiedono al dire del Barberino in materia di formazione dell'uomo.

Il radicamento dell'uomo nel mondo, la conquista di una fama non effimera deve avvenire dunque all'ombra di questi modelli ideali che le immagini del trattato si incaricano di mostrare. E risulta quanto mai ovvio riconoscere qui uno dei segnali più forti di aggancio (del progetto barberiniano di visualizzazione di un percorso cognitivo puntato all'affermazione di sé nel mondo) a quel che in parallelo - ma con esiti di senso opposto, tutti interni a valori profani e di mundanitas - aveva testualizzato anche Brunetto Latini col suo Tresor, emblema di un'“eternità" conquistabile con un impegno tutto e solo pragmatico

34 Per gli esempi di valore predicativo superiore riconosciuti alle immagini rispetto alle parole cfr. gli esempi portati fra molti altri da MacLaren, Shaping the Self, pp. 75-95.

35 Ho ricostruito questa prospettiva, fondamentale per intendere il processo cognitivo che sta alla base dell'emancipazione delle immagini dal cosiddetto imperialismo gotico della parola, in diversi saggi, a partire da Ciccuto, Il novelliere, poi approdati sul versante di un riscontro teorico interno all'operare dantesco nella relazione Agostino e Bonaventura: per una teologia dellimmagine dantesca, presentata al Convegno internazionale Immagine poetica, immaginazione: Dante e la cultura medioevale, svoltosi presso la Società Dantesca Italiana in Firenze nell'aprile 2017, e ora pubblicata in "Letteratura e Arte" 16 (2018).

36 Molto puntuale e convincente l'interpretazione fornita a riguardo in Panzera, Francesco da Barberino, pp. 195-197; 203-204. 
nell'esistenza (come Dante in persona avrà modo di ricordare e sigillare con incomparabile chiarezza nell'episodio dell'incontro con l'antico suo maestro nell'area infernale riservata a chi agì contro la Natura divina del cosmo e poté perciò essere rappresentato come un peccatore per antonomasia "contro natura"). ${ }^{37} \mathrm{Al}$ lontanarsi dalla caducità e dall'inconstantia dei fortuiti fatti umani - sia Giotto che Brunetto del resto ebbero modo di far coincidere il tema della incostante $m u$ tabilitas del mondo degli uomini con una centration sul tema di Fortuna, che pure Dante viene a utilizzare (oltretutto con vistosi riscontri "astrologici”) al fine precipuo di definire secondo la sua prospettiva l'impegno tutto mondano del retore fiorentino ${ }^{38}$ - diventò in effetti fulcro della riflessione per la quale lo stesso

37 Per la lettura di questo episodio di Inf. XV, il progetto culturale brunettiano di sola affermazione dell'uomo nell'esistente e l'eco che ne risultò nel contesto culturale primo-trecentesco cfr. Ciccuto, Premessa, pp. 141-ss., nonché Ciccuto, Tradizioni illustrative. La successiva lettura del canto XVI infernale, orientata nel medesimo senso (Ciccuto, Cortesia e dismisura), viene a rappresentare una sorta di ribaltamento della prospettiva brunettiana dichiarata e percorsa nel canto XV all'insegna del conseguimento di un'eternità vincolata appunto all'impegno esclusivo negli affari terreni: in giunzione per di più con la proposta di riflessione attorno al «ver ch'ha faccia di menzogna» sulla quale Dante fonderà un suo primo ma ben convinto allontanamento dalle vane fictiones della poesia classica.

38 Romano, Giotto e la nuova pittura, pp. 15-17, e Ciccuto, Premessa, passim. Se dunque Barberino ha modo di citare in esplicito il Tresor (cfr. Panzera, Francesco da Barberino, p. 185) rivelandone una conoscenza puntuale, va aggiunto che nella copia laurenziana di quel testo brunettiano, ms. Pluteo 42 19, troviamo all'opera vistosamente quel Maestro Daddesco cui si devono accostamenti forti al discorso per figuras di Francesco, nonché aggancio preciso all'area esecutiva del Bildercodex che caratterizzò l'attività tutta della bottega pacinesca, anche nelle vicinanze dell'operare di Convenevole da Prato (su tutto questo cfr. Pasut 2008, pp. 54-55 e Sciacca, Pacino di Bonaguida, pp. 37-38, nonché Smout, Sprechen in Bildern, passim): saranno dunque le affinità di basi disegnative poi segnalate sulle carte del Barb. lat. 4076, le coerenze pacinesche che per prodotti sia brunettiani sia barberiniani ha rilevato Pasut, Pacino di Bonaguida, pp. 406-408; più tardi la concordanza di questi elementi coi disegni a penna e acquarellati del Messale di San Pier Maggiore - Pasut 2008, p. 55 e Labriola, L'eredità di Giotto, pp. 68-69 - su su fino a quanto accertato da Sciacca, Pacino di Bonaguida, pp. 199-200 in merito alla cosiddetta Bibbia Trivulziana. Per i rapporti ravvicinati tra la figurazione dei Documenti e l'esemplare laurenziano del Tresor cfr. comunque gli importanti avvisi di Roux, Mondes en miniatures, pp. 255-259 (e per la centralità della rappresentazione di Constantia, pp. 257-258). Voglio allora ricordare, sulla scia di quanto accertato in Panzera, Francesco da Barberino, pp. 61-63, che in questo giro di cultura trovava luogo e prendeva significati aggiuntivi il coinvolgimento di un personaggio quale Andrea de' Mozzi - figurante come ognun sa anche negli interstizi del canto infernale di Brunetto - nella vicenda del vescovo Antonio degli Orsi, della villa episcopale di Montughi e della chiesa di San Miniato al Monte, dove la presenza in antico di un mosaico di soggetto astrologico nonché il peso dell'inserimento dantesco di quel complesso nel suo proprio discutere attorno ai temi della gloria mondana e della fortuna, tanto per dire, nel canto XII del Purgatorio, si teneva accosto al contesto 
Barberino venne a generare la funzione primaria delle sue Bildbegriffe che consisteva, in sostanza e in posizione di vistoso equilibrio dentro l'assetto teorico dei Documenti, nell'additamento del vizio di superbia quale fondamento a più vasto argomentare - che sarà vieppiù specificamente dantesco -, quello cioè intrecciato al tema della fama o gloria mondana, già ben corrente se non addirittura decisivo in quel contesto padovano che aveva visto lo Scrovegni, accusato di puntare ad vanam gloriam, correre ai ripari in ispecie con l'edificazione della Cappella all'Arena e la commissione dei suoi cicli figurati all'operare del genio giottesco. ${ }^{39}$

La già ricordata registrazione memoriale dell'icona giottesca dell'Invidia nel commento barberiniano ( $D A$ [Egidi], II, p. 165) ${ }^{40}$ può agire da apripista sulla via di una comprensione dello stretto quanto sorprendente rapporto che resiste individuabile fra il significato generativo dell'intera riflessione di Francesco, affidata al filo rosso del concetto di fama terrena all'interno dei Documenti, e l'analogon realizzato da Dante nella mirabile sequenza sul peccato di superbia inteso alla gloria terrena nei canti X, XI e XII del Purgatorio, a loro volta portatori di un articolato ragionare sulla funzione congiunta di immagini e di parole che pare avere uno dei suoi apici proprio nella mise en scène di artisti conosciuti, pur in differente grado di approssimazione, da entrambi i poeti, e cioè Cimabue e Giotto (oltre beninteso a Oderisi e al cosiddetto Franco bolognese).

Se solo riflettiamo sul collegamento che il Barberino esplicita fra discorso sulla gloria umana, peccato di superbia e insegnamenti avversi ai loro modi di apparenza affidati alla funzione collaborativa di pitture e scritture, ${ }^{41}$ non è diffi-

\footnotetext{
del «visibile parlare» oltreché all'immagine di una chiesa che appunto aveva posto Firenze sotto il giogo di valori di incostanza o impermanenza in chiave anti-provvidenziale e di indubitabile declinazione astrologica. Per come poi Barberino ebbe a organizzare la dorsale ragionativa di parte dei Documenti fra la centralità dell'iconografia e del ruolo di Giustizia (o misuraequilibrio che dir si voglia) e il confronto con la collocazione in quell'opera di una tutta giottesca figura di Inconstantia, cfr. Romano, Giotto e la nuova pittura, pp. 13-17.

39 Gardner, Giotto, p. 65.

40 Per un'idea congiuntiva fra invenzione di Giotto per quella figura e trattamento dantesco del relativo peccato entro i canti XIII-XV del Purgatorio valgano le osservazioni e le ipotesi avanzate da Romano, La O di Giotto, pp. 221-222.

41 Della "fama" da subito implicata a tracce forti così del discorso sul vizio di superbia come a elementi di visività introdotti nella grana del testo potremmo dare qui numerosi esempi fra i più eloquenti; mi limito tuttavia all'indicazione dei luoghi più incisivi, quali quelli in $D A$ (Egidi), I, pp. 52, 55, 79, 91, 149, 254-261 giusto sul peccato di superbia specialmente connesso a fattori di esibizione visiva; II, 95 sulla gloria “verace” e non di apparenza, secondo un ragionamento esteso più avanti - II, 295 - all'immagine dei libri di modesta confezione e dunque più validi e degni di gloria di quelli impostati su fini di ornamento o di scrivere "bello" e superficiale; VI, 250-253 e 268-273 interamente versati sulla definizione dei rapporti tra vanagloria e apparenze.
} 
cile intendere quanto le proposte della didassi barberiniana si possano ritenere vicine - se non addirittura anticipatrici - rispetto al grande bacino argomentativo entro cui poi lo stesso Dante Alighieri ebbe a calare la sua propria condanna della praesumptio alios superandi. In quei canti si venne a presentare di fatto una testualità sopra le righe, accresciuta per via di enargeia a ogni pie' sospinto, con vistosi processi di amplificatio, vari loci agentes in funzione di apostrofi, esclamazioni, invettive, tutta una retorica di exsuscitatio l'avrebbero chiamata gli antichi, piegata peraltro all'effetto di un accentuato coinvolgimento emotivo come è nella tradizione degli epigrammi ecfrastici dell'Anthologia greca e latina che descrivono al passante le opere d'arte sul cammino, ${ }^{42}$ fino a parole che il poeta si incarica di presentare come entità quasi autonome - ad esempio nello straordinario segmento dei vv. 46-51 del canto XI; direi nella sostanza al fine di dare l'idea di notevoli risorse tecniche tipiche del dire classico o degli antichi in genere, rimaste tuttavia limitate a un esercizio di enargeia fine a se stesso, incapace di dare vita o di sviluppare quello che sarà l'obiettivo più vero di Dante con la realizzazione degli esempi "divini” e dunque un superiore senso di morale attiva, sotto ogni profilo distante da forme di "superbia intellettuale" di cui lo stesso poeta si è reso reo nel corso della sua originaria imitazione, solo tecnicamente fervida, dei classici latini.

Si vede bene peraltro che la stessa struttura narrativa di questi canti risulta allestita in modo da convergere prima verso l'immagine dell'Annunciazione ${ }^{43} \mathrm{e}$ a seguire verso la figurazione dell'episodio di Traiano e della vedova, inteso questo quale punto di forte esibizionismo della verbalità o "evidenza” della parola; per quanto ci si possa accorgere agevolmente che una funzione assai più dirimente e speciale avrà, a livello però strutturale, quella strana inserzione della recita del Pater noster nella forma di preghiera collettiva, apertura totalizzante di un testo dinamizzato all'estremo, che avrà il compito di equilibrare e finanche contrastare la funzione affidata all'immagine delle cariatidi "umane" e pure all'acrostico inscritto alla preghiera: sculture quelle di creazione terrestre, circunscritte a differenza del Dio non circunscritto del Pater noster, mentre l'acrostico nella parola VOM verrà ad assumere i valori tutti di ogni artificio verbale, di qualsivoglia figura di ostentazione dell'arte umana la cui superficiale

42 Su questo si può vedere anche Battistini, La «speranza de l'altezza», pp. 101-105.

43 Sull'iconografia dell'Annunciazione connessa ad alcune funzioni quaresimali legate al principio di liberazione e penitenza del peccato di superbia, nonché sul convergere di questa immagine in particolare su circostanza e data di inaugurazione della Cappella all'Arena si trovano parole importanti in Romano, Giotto e la nuova pittura, pp. 26-28. 
varietas si vede restare perfettamente in linea con l'eloquio iperespressivo dei superbia di questa zona. ${ }^{44}$

In quest'area dantesca siamo alle viste di una sorta di esibizionismo della verbalità e dei suoi registri di dinamica espressione potenziata al massimo dall'additamento a valori figurali, non ultimo obiettivo collegato quello di dare l'impressione di uno scorrimento dei personaggi in itinere davanti alle figurazioni purgatoriali, fors'anche presi nel giro di una partecipazione emotiva o reazione emozionale che dir si voglia rispetto a questa concreta azione. A uno dei culmini di questi procedimenti costruttivi del canto starà dunque il discorso di Oderisi, insieme figurato e performativo, incrociato a un autentico mosaico di imagines agentes, quasi che il miniatore dovesse porsi a campione del valore transitorio di colores e potenze dell'enargeia; di una parola allora che sarà l'integrale della contingenza terrena, luogo precipuo di mutevolezza e peribilità scambiate ora per "gloria della lingua" ora per valori di eternità conquistati dal fare umano, e insomma destinati a finir superati dal sacrato dire per bocca di Adamo in Paradiso XXVI. ${ }^{45}$ Quando poi scatterà una delle espressioni sulle quali si puntella il “colorato” parlare di Oderisi, «e l'uno e l'altro caccerà del nido», otterremo un altro segnale forte circa un argomentare di Dante costruito proprio sul concetto dell'elevazione intellettuale del poeta solo avvinta alle pochezze delle passioni umane, quindi alle peribili effervescenze dei raffinati esercizi retorici, dell'enargeia verbale non ancora aperta alla ben più densa e profonda pluralità del trasumanar di cui le immagini possono fornire sufficiente rilievo.

Mi pare addirittura evidente come Dante venga promuovendo, in quest'area della sua narrazione purgatoriale, un modello di scrittura che echeggia e specchia quella epigrammatico-funeraria, della cui ipertrofia retorica è capace di mettere a partito tutte le possibilità: abbondanza di dettagli, animazione delle figure, interazione col lettore, relazioni plurisensoriali, appelli al lettore-osservatore, fissazione di un'esperienza percettiva davanti agli occhi del riguardante, descri-

44 Il valore di potenziamento magico-rituale che l'insieme di segno grafico e lettere assume nel caso della composizione di un acrostico può essere chiarito in base alle osservazioni di Agosti, Saxa loquuntur?, pp. 179-180. Ma cfr. per esso quanto ho già indicato in Ciccuto, Saxa loquuntur, p. 155 e nota 16.

45 Quanto al discorso insieme figurato e performativo di Oderisi, mosaico di autentiche imagines agentes a disposizione di un personaggio che quasi figura da campione dei valori transitori di colores e di enargeia, si veda il bel saggio di Marini, «La gloria de la lingua». Peraltro lo stile "visibilistico" del discorso del miniatore aveva già attirato l'attenzione di Gorni, Guittone e Dante, mentre per l'evoluzione del dire poetico dantesco dalle figure del sublime retorico alle "sostanze" della "lingua sacrata" di Adamo nell'Eden e nel passaggio dei canti XXV-XXVI del Paradiso mi si permetta di rinviare a Ciccuto, La parola che salva. 
zione di cose che prima di tutto si debbano vedere. ${ }^{46}$ Incorporate le funzioni ostensive delle figure all'interno della scrittura poetica, si attiva una sorta di multisensorialità per via di un congegno verbale iper-retoricizzato quale fu per Dante quello dei classici, utile non solo a trasmettere la descrizione di un'opera in sé bensì soprattutto le modalità di un vedere l'oggetto artistico; ${ }^{47} \mathrm{ci}$ si allontana così dalla pura mimesi per attingere a risorse di sicura complicanza figurale e insieme si tenta una mise en langage del visuale convocando tutti i sensi di un lettore diventato ora anche osservatore. E va riconosciuto che gli ingredienti classici di questa operazione sul linguaggio ci sono tutti: dalla scenografia oratoria al lessico enfaticamente visivo, dal messaggio di pietra (Steinepigramme) alla figura del wandering poet, rapsoda e passer-by, sino a performances enunciative di grande effetto come il tradizionale dialogo con la stele, ${ }^{48}$ alla lettura infine ad alta voce da "superbia enunciativa" e alle indicazioni circa i movimenti vivaci del discorso, di un parlare che si fa vita visibile sotto gli occhi del viaggiatore-osservatore (da cui procede anche l'inserzione di espressioni ricercate, di tessere lessicali preziose che attirano, talvolta veri e propri oracoli teologici dal potere magico-rituale indipendente dal testo, verso la voce della divinità, e che Barberino aveva invero condannato come valori d'apparenza al pari di ciò che rende lo "specchio magico" a chi crede alle finzioni rappresentative). ${ }^{49}$

$\hat{E}$ in questo modo che viene eseguita e additata da Dante una forma di superbia dell'arte umana, fondata su una sublime ma vuota ricercatezza, su uno stare sopra le linee del discorso ordinario che è però solo un effet de surface rispetto all'arte divina (che dà vero movimento all'inanimato, né si perde in sterili gare per primeggiare verbalmente ma sola offre immagini perfette, aderenti a superiori idealità). ${ }^{50}$ Certo il compito che Dante si assume non può se non coincidere con un gareggiare coi classici usando i loro stessi strumenti, appunto verbali; ma per procedere oltre, per passare a più alto grado di creatività, se vogliamo identificabile nel modello esposto del Pater noster - e non dunque dall'acrostico, destinato a restare il limite del creare verbale terreno rispetto alle potenzialità del visivo e del visibile. ${ }^{51}$ Magari l'uomo-poeta, come Barberino,

46 Importanti i riscontri in merito di Debiais, La vue des autres, pp. 395-396.

47 Debiais, La vue des autres, pp. 397-398.

$48 \mathrm{Si}$ tratta della situazione nella quale «la performance della lettura perpetua la vita del defunto» (Agosti, Saxa loquuntur?, p. 166).

49 Anche questo d'altronde può essere il contesto dal quale può essersi generata l'idea e l'immagine dell'acrostico: cfr. Agosti, Saxa loquuntur?, pp. 173-179.

50 Per il concetto del primeggiare, inordinata praesumptio alios superandi, sul quale ha le sue radici lo sviluppo comune di superbia, invidia e avarizia, cfr. Ragni, Canto X, pp. 269-270.

51 Di fatto è nella preghiera dettata dal Cristo in persona che possiamo riconoscere un esempio di "scrittura paradisiaca", nella quale la superiorità dei modi transumptivi arricchiti dalle ri- 
potrà diventare campione nel far coincidere efficacemente verbalità e visività, ma mai si alzerà al parlare visibile divino che reca connaturato in sé il trasumanar - che è, si badi, il più autentico fine di tutti $i$ disii, cioè a dire ciò a cui tende il caos di sensazioni che può essere evocato da un'opera d'arte comunque mirabile. E insomma, invece che una rappresentazione tecnicamente più che perfetta, "energizzata" e raffinata allo spasimo, capace allora di movimentare i disii che improntano l'eccellenza terrena e mimetica assieme alla superbia, Dante suggerisce in alternativa e avanzamento di prospettiva il puntare all'attivazione della phantasia tramite la tragedizzazione dello stile o i modi transumptivi tendenti al sublime dell'espressione, senza contentarsi dei risultati di pura efficacia tecnica di questi stessi modi transumptivi - buoni secondo Dante per gente «della vista della mente inferma» specie se contenta di soddisfare la sola concupiscentia oculorum $^{52}$ - ma andando a cogliere quel quid ineffabile che solo alcune fra le immagini prodotte dall'uomo sono in grado di adombrare e suggerire: appreso a un veder maggio, naturalmente, ben al di là di ogni capacità di abbellimento, e che sapremo essere più avanti nel testo del poema la purezza infantile e atavica del linguaggio adamitico contenente ogni senso e figura, il limite di silenzio e di ineffabilità ma appunto e finalmente cosa vivente esso stesso. ${ }^{53}$

Ebbene Francesco da Barberino non solo fu uno dei primi "movimentatori" di funzioni che ai dicta vulgaria avrebbero portato la forza espressiva, l'“energia” diremmo con termine tecnico, delle immagini dipinte e in ispecie di quelle arrivate sui fogli dei Documenti d'Amore, delle didascalie e degli inscripta associati alle scene figurate $;{ }^{54} \mathrm{fu}$ anche e specialmente il responsabile di una nuova strategia del guardare ai segni del vivere, fossero essi verbali o iconici: davanti ad essi l'homo novus, temprato alla resa visibile dei valori più sacri di virtù frammisti alle apparenze terrene, avrebbe avuto i mezzi sufficienti a dare un ordine a ogni

sorse iconiche del logos la fa da padrona nella forma di una verbalità complessa, sola capace di esprimere l'avvicinamento al «fine di tutt'i disii» (Par. XXXIII, 46). Di rilievo su questo la riflessione in Lombardo, Dante et l'ekphrasis, pp. 113-115.

52 Lombardo, Dante et l'ekphrasis, pp. 111-113. L'importanza delle impressioni cognitive necessarie al procedere della phantasia verso una superiore condizione espressiva è messa in luce da Squire, Reading a View, pp. 78-80.

53 Come scrive Lombardo, Dante et l'ekphrasis, p. 117: «l'extase divine atteinte par Dante se donne donc sous la forme d'une mimésis du silence reproposant, au-delà du langage, l'inhabilité verbale d'une vie qui vient de s'éveiller, d'une existance encore arrêtée en deçà du langage». E cfr. in aggiunta Ciccuto, «Trattando l'ombre».

54 Panzera, Francesco da Barberino, pp. 68-69; 73-74. E per il debito verso le iscrizioni giottesche cfr. Goldin Folena, Dall'“Officiolo”, pp. 109-116. 
progetto cognitivo o finanche educativo che si proponesse di recuperare laicamente, dentro il vivere, l'antica capacità di esprimere sia in immagini sia in parole tutte le più alte idealità del cosmo cristiano.

\section{Bibliografia}

\section{Fonti}

Geri d'Arezzo, Lettere e dialogo d'amore, introduzione, traduzione e testo critico di C. Cenni, Ospedaletto ( $\mathrm{Pisa}) 2010$.

Guittone d'Arezzo, Del carnale amore, a cura di R. Capelli, Roma 2007.

Poeti del dolce stil nuovo, a cura di M. Marti, Firenze 1969.

\section{Studi critici}

G. Agosti, Saxa loquuntur? Epigrammi epigrafici e diffusione della paideia nell'Oriente tardoantico, in «Antiquité tardive», 18 (2010), pp. 163-180.

M. Amri-Kilani, Connaissance sensitive et rhétorique chez Alain de Lille, in Alain de Lille, le docteur universel. Philosophie, théologie et littérature au XII siècle, Turnhout 2005, pp. 191-215.

D. Banzato, L'impronta di Giotto e lo sviluppo della pittura del Trecento a Padova, in Giotto e il Trecento. «ll più Sovrano Maestro stato in dipintura», catalogo a cura di A. Tomei, Milano 2009, pp. 143-156.

A. Battistini, La «speranza de l'altezza». La retorica patetica in Purgatorio, XII, in «L'Alighieri», 21 (2003), pp. 95-108.

H. Belting, Das Bild als Text: Wandmalerei und Literatur im Zeitalter Dantes, in Malerei und Stadtkultur in der Dantezeit: Die Argumentation der Bilder, herausgegeben von H. Belting, D. Blume, München 1989, pp. 23-64.

S. Bertelli, L'“Officiolo": il manoscritto, in Officiolum di Francesco da Barberino Commentario all'edizione in fac-simile, a cura di S. Bertelli et al., Roma 2016, pp. 21-36.

D. Blume, Michael Scot, Giotto and the Construction of New Images of the Planets, in Images of the Pagan Gods, Papers of a Conference in Memory of Jean Seznec, eds. R. Duits, F. Quiviger, London-Torino 2009, pp. 129-150.

D. Blume, M. Haffner, W. Metzger, Sternbilder des Mittelalters und der Renaissance, Berlin 2016.

S. Carapezza, Funzioni digressive nella didattica medievale. Psychomachia, Anticlaudianus $e$ L'Intelligenza, in Uso, riuso e abuso dei classici, a cura di M. Gioseffi, Milano 2012, pp. 105-120.

M. Ciccuto, Cortesia e dismisura: i compagni avversi di Brunetto Latini (Inferno, XVI), in «Lectura Dantis Lupiensis», 2 (2014), pp. 117-131. 
M. Ciccuto, Fatti romani del Tito Livio Colonna, in Reliquiarium servator. Il manoscritto Parigino latino 5690 e la storia di Roma nel Livio dei Colonna e di Francesco Petrarca, a cura di M. Ciccuto, G. Crevatin, E. Fenzi, Pisa 2012, pp. 11-58.

M. Ciccuto, Figure d'artista. La nascita delle immagini alle origini della letteratura, Fiesole 1996.

M. Ciccuto, Figure di Petrarca (Giotto, Simone Martini, Franco bolognese), Napoli 1991.

M. Ciccuto, Francesco da Barberino: un pioniere del "Bildercodex" tra forme del gotico cortese e icone della civiltà comunale, in «Letteratura \& Arte», 9 (2011), pp. 83-95.

M. Ciccuto, Icone della parola. Immagine e scrittura nella letteratura delle origini, Modena 1995.

M. Ciccuto, Il novelliere "en artiste": strategie della dissimiglianza fra Boccaccio e Bandello, in L'immagine del testo. Episodi di cultura figurativa nella letteratura italiana, Roma 1990, pp. 113-156.

M. Ciccuto, La parola che salva: per un ritratto di Dante filosofo del linguaggio, in «Quaderns d'Italià», 18 (2013), pp. 65-78.

M. Ciccuto, Premessa al Tesoretto di Brunetto Latini, in Il restauro de "L'Intelligenza» e altri studi dugenteschi, Pisa 1985, pp. 141-158.

M. Ciccuto, Saxa loquuntur. Aspetti dell'evidentia nella retorica visiva di Dante, in Dante e la retorica, a cura di L. Marcozzi, Ravenna 2017, pp. 151-166.

M. Ciccuto, Tradizioni illustrative attorno a «Tresor» $e$ «Tesoretto», in A scuola con ser Brunetto. Indagini sulla ricezione di Brunetto Latini dal Medioevo al Rinascimento, Atti del Convegno internazionale (Basilea, 8-10 giugno 2006), a cura di I. Maffia Scariati, Firenze 2008, pp. 3-12.

M. Ciccuto, «Trattando l'ombre come cosa salda». Forme visive della "dolcezza" di Stazio nel Purgatorio dantesco, in Studi sul canone letterario del Trecento. Per Michelangelo Picone, a cura di J. Bartuschat, L. Rossi, Ravenna 2003, pp. 57-66.

M. Ciccuto, Un'antica canzone di Giotto e i pittori di Boccaccio. Nascita dell'identità artistica, in «Intersezioni», 16/3 (1996), pp. 403-416.

V. Debiais, La vue des autres. L'ekphrasis au risque de la littérature médiolatine, in "Cahiers de civilisation médiévale», 55 (2012), pp. 393-404.

M.L. Fuehrer, The Cosmological Implications of the Psychomachia in Alan of Lille's Anticlaudianus, in «Studies in Philology», 77/4 (1980), pp. 344-353.

P. Galand, Les «beaux» signes. Un «locus amoenus» d'Alain de Lille, in «Littérature», 74 (1989), pp. 27-46.

J. Gardner, Giotto: «First of the Moderns» or Last of the Ancients?, in «Wiener Jahrbuch für Kunstgeschichte», 44/1 (1991), pp. 63-78.

D. Goldin Folena, Dall'l "Officiolo" ai "Documenti d'Amore" all'“Officiolo": Francesco da Barberino illustratore, in Officiolum di Francesco da Barberino. Commentario all'edizione in fac-simile, a cura di S. Bertelli et al., Roma 2016, pp. 105-159.

G. Gorni, Guittone e Dante, in Guittone d'Arezzo nel settimo centenario della morte, Atti del Convegno internazionale di Arezzo (22-24 aprile 1994), a cura di M. Picone, Firenze 2015, pp.309-335.

C. Gross, The Cosmology of Rhetoric in the Early Troubadour Lyric, in «Rhetorica», 9/1 (1991), pp. 39-53.

C. Harding, Speaking in Pictures: Reading, Memory and Interpretation in Francesco da Barberino's Advice to Women in his Reggimento e costumi di donna, in «Racar: revue d'art canadienne / Canadian Art Review», 36/1 (2011), pp. 29-40. 
A. Hoffmann, Die Bibel von Gerona und ihr Meister, Berlin-München 2013.

A. Labriola, L'eredità di Giotto nella miniatura fiorentina, in L'eredità di Giotto Arte a Firenze 1340-1375, Firenze 2008, pp. 67-76.

G. Lombardo, Dante et l'ekphrasis sublime. Quelques remarques sur le «visibile parlare» (Purg. X, 95), in Les arts - quand il se rencontrent, éd. J. Pigeaud, Rennes 2009, pp. 99-119.

S. MacLaren, Shaping the Self in the Image of Virtue: Francesco da Barberino's “I Documenti d'Amore", in Image and Imagination of the Religious Self in Late Medieval and Early Modern Europe, eds. N. Falkenburg, S. Melon, T.M. Richardson, Turnhout 2007, pp. 71-95.

G. Mariani Canova, Il poeta e la sua immagine: il contributo della miniatura alla localizzazione e alla datazione dei canzonieri provenzali AIK e $N$, in I trovatori nel Veneto e a Venezia, Atti del Convegno internazionale di Venezia (28-31 ottobre 2004), a cura di G. Lachin, Roma-Padova 2008, pp. 47-76.

G. Mariani Canova, La Cattedrale di Padova e i suoi manoscritti miniati. Libri della liturgia e della cultura dal Medioevo al primo Rinascimento, in I manoscritti miniati della Biblioteca Capitolare di Padova, I. I manoscritti medievali e proto rinascimentali della Chiesa padovana e di altra provenienza, a cura di G. Mariani Canova, M. Minazzato, F. Toniolo, Padova 2014, pp. 1-59.

G. Mariani Canova, L'“Officiolo” di Francesco da Barberino e le sue figure: libro di devozione, diario dell'anima, palestra di stile, in Officiolum di Francesco da Barberino. Commentario all'edizione in fac-simile, a cura di S. Bertelli et al., Roma 2016, pp. 63-104.

P. Marini, «La gloria de la lingua» nel trittico dei superbi. Considerazioni sul nodo arte-onoresuperbia-umiltà nella Commedia, in «Italianistica», 36/3 (2007), pp. 65-88.

G. Marruzzo, Composizione e significato de "I documenti d'Amore" di Francesco da Barberino, in «Giornale Italiano di Filologia», 5/3 (1974), pp. 217-251.

A.J. Minnis, Amor and Auctoritas in the Self-Commentary of Dante and Francesco da Barberino, in «Poetica», 32 (1990), pp. 25-42.

V. Nardi, Le illustrazioni dei “Documenti d'Amore” di Francesco da Barberino, in «Ricerche di storia dell'arte», 49 (1993), pp. 75-92.

M.C. Panzera, Francesco da Barberino tra Andrea Cappellano e Averroè. Poesia, immagini, profetismo, Alessandria 2016.

F. Pasut, Pacino di Bonaguida e le miniature della Divina Commedia: un percorso tra codici poco noti, in Da Giotto a Botticelli. Pittura fiorentina tra Gotico e Rinascimento, Atti del convegno internazionale (Firenze, 20-21 maggio 2005), a cura di F. Pasut, J. Tripps, Firenze 2006, pp. 41-62.

A. Pegoretti, Indagine su un codice dantesco. La «Commedia» Egerton 943 della British Library, Ghezzano (Pisa) 2014.

C. Ponchia, Le fonti illustrative delle miniature del Dante Egerton, in Il Manoscritto Egerton 943 Dante Alighieri Commedia. Saggi e commenti, a cura di M. Santagata, Roma 2015, pp. 109-128.

S. Prandi, Teologia come pittura: Alain de Lille e Dante (Purg. X-XII), in La parola e l'immagine Studi in onore di Gianni Venturi, vol. I, a cura di M. Ariani et al., Firenze 2011, pp. 99-116.

E. Ragni, Canto X. Umiltà, superbia e "visibile parlare", in Lectura Dantis Romana Cento canti per cento anni, vol. II, Purgatorio. Canti I-XVII>, a cura di E. Malato, A. Mazzucchi, Roma 2014, pp. 266-297. 
V. Rodrigues, Nature et connaissance de la nature dans le Sermo de sphaera intelligibili et dans les Glosae super Trismegistum, in Alain de Lille, le docteur universel. Philosophie, théologie et littérature au XII siècle, Turnhout 2005, pp. 169-190.

S. Romano, Giotto e la nuova pittura. Immagine, parola e tecnica nel primo Trecento italiano, in Il secolo di Giotto nel Veneto, a cura di G. Valenzano, F. Toniolo, Venezia 2007, pp. 7-46.

S. Romano, La O di Giotto, Milano 2008.

F. Rouillé, L'hymne «Omnis mundi creatura», une miniature de l'Anticlaudien d'Alain de Lille (XII S.)?, in «Camenae», 1 (2007), pp. 1-17.

B. Roux, Mondes en miniatures. L'iconographie du Livre du Trésor de Brunetto Latini, Genève 2009.

C. Sciacca, Pacino di Bonaguida and his Workshop, in Florence at the Dawn of the Renaissance. Painting and Illumination, 1300-1350, ed. C. Sciacca, Los Angeles 2012, pp. 285-303.

B. Schirg, "In bivio". Zur Lebenswegentscheidung als Motiv frühhumanistischer Selbstdarstellung bei Geri d'Arezzo und Francesco Petrarca, in «Studi medievali», 55 (2014), pp. 299-340.

C. Smout, Sprechen in Bildern - Sprechen über Bilder. Die allegorischen Ikonotexte in den Regia Carmina des Convenevole da Prato, Köln 2017.

M. Squire, Reading a View: Poem and Picture in the Greek Anthology, in «Ramus», 39/2 (2010), pp. 73-103.

A. Stolte, Der Maestro di Gherarduccio kopiert Giotto. Zur Rezeption der Arena-Fresken in der oberitalienischen Buchmalerei zu Beginn des 14. Jahrhunderts, in «Mitteilungen des Kunsthistorischen Institutes in Florenz», 40/1-2 (1996), pp. 2-41.

A. Stolte, Frühe Miniaturen zu Dantes “Divina Commedia”. Der Codex Egerton 943 der British Library, Münster 1998.

F. Toniolo, Il libro miniato a Padova nel Trecento, in Il secolo di Giotto nel Veneto, a cura di G. Valenzano, F. Toniolo, Venezia 2007, pp. 107-152.

F. Toniolo, Il Maestro degli Antifonari di Padova miniatore del Dante Egerton, in Il Manoscritto Egerton 943 Dante Alighieri Commedia. Saggi e commenti, a cura di M. Santagata, Roma 2015, pp. 89-108.

F. Toniolo, Il Maestro degli Antifonari di Padova. Prassi e modelli, in Medioevo. Le officine, Atti del Convegno internazionale di Parma (22-27 settembre 2009), Milano 2010, pp. 549-562.

A. Vasiliu, L'économie de l'image dans la sphère intelligible (sur un sermon d'Alain de Lille), in «Cahiers de civilisation médiévale», 41 (1998), pp. 257-279. 